\title{
Article
}

\section{Simulation Studies on High-Field EPR Spectra of Lipid Spin Labels in Cholesterol-Containing Membranes}

Vsevolod A. Livshits, Dieter Kurad, and Derek Marsh

J. Phys. Chem. B, 2004, 108 (27), 9403-9411• DOI: 10.1021/jp035915p • Publication Date (Web): 30 March 2004

Downloaded from http://pubs.acs.org on March 23, 2009

\section{More About This Article}

Additional resources and features associated with this article are available within the HTML version:

- $\quad$ Supporting Information

- $\quad$ Access to high resolution figures

- $\quad$ Links to articles and content related to this article

- $\quad$ Copyright permission to reproduce figures and/or text from this article

\section{View the Full Text HTML}

\section{ACS Publications}




\title{
Simulation Studies on High-Field EPR Spectra of Lipid Spin Labels in Cholesterol-Containing Membranes ${ }^{\dagger}$
}

\author{
Vsevolod A. Livshits, ${ }^{\S}$ Dieter Kurad, ${ }^{\ddagger}$ and Derek Marsh*, \\ Max-Planck-Institut für biophysikalische Chemie, Abteilung Spektroskopie, 37077 Göttingen, Germany, and \\ Centre of Photochemistry, Russian Academy of Sciences, 117427 Moscow, Russian Federation
}

Received: July 3, 2003; In Final Form: November 14, 2003

\begin{abstract}
Simulations are presented of 94-GHz and 9-GHz EPR spectra from phospholipid probes spin-labeled at the $\mathrm{C} 4$ to $\mathrm{C} 14$ positions of the $s n-2$ chain in liquid-ordered membranes of dimyristoyl phosphatidylcholine that contain $40 \mathrm{~mol} \%$ cholesterol. Spectra at $94 \mathrm{GHz}$ can be simulated adequately by motional narrowing theory. The latter accounts better for averaging of the $g_{x x} / g_{y y}$ canonical features, with a restricted rotation about the $z$ axis, than do slow-motional descriptions (Brownian and strong-jump) of unrestricted $\phi$ rotation. Polaritycorrected $g$ tensors are required for the high-field simulations. These are obtained from measurements at low temperature $\left(-100^{\circ} \mathrm{C}\right.$ ) by comparing the polarity profile with spin-label position, $n$, that is based on $g_{0}$ (i.e., the trace of the $g$ tensor) with that established from simulations of experimental spectra at the measurement temperature of $+30^{\circ} \mathrm{C}$. Model simulations for Brownian rotational diffusion indicate that the $A_{z z}$ element of the ${ }^{14} \mathrm{~N}$ hyperfine splitting of the $94-\mathrm{GHz}$ spectra is relatively insensitive to slow off-axial diffusion. This result is used to derive spin Hamiltonian tensors that are partially averaged by the fast motional component, from the high-field spectra. These are then used in the stochastic Liouville equation to obtain anisotropic parameters of the slow motional component that is evident in the experimental 9-GHz spectra. The rapid diffusional component is attributed to segmental motion of the lipid chains, and the slow diffusional component to angular fluctuations of the chain axis. The latter are not constant throughout the length of the chain, but increase in intensity towards the terminal methyl region.
\end{abstract}

\section{Introduction}

The applications of high-field EPR spectroscopy to the study of nitroxyl spin labels in biological systems are growing rapidly (for reviews see refs 1-3). Spectral simulations form an integral part of this development (see, e.g., ref 4). Biological membranes, which are intrinsically anisotropic but dynamic structures, constitute a particularly fruitful area for spin-label EPR at high field. Previously, we found that $94-\mathrm{GHz}$ EPR spectra of spinlabeled lipids in cholesterol-containing membranes are better simulated by a rapid motional model in which $\phi$ rotation around the lipid chain axis is restricted than by a slow-motional strongjump formalism with unrestricted $\phi$ rotation. ${ }^{5}$ Recently, by using a multifrequency approach, Lou et al. ${ }^{6}$ showed that high-field spectra (at $250 \mathrm{GHz}$ ) are sensitive only to rapid local motions for a spin-label close to the terminal methyl group of the lipid chains. Low-field, 9-GHz EPR spectra of the same spin label were found to be sensitive additionally to slow overall rotational diffusion of the lipid chain segments.

Here we develop our simulation studies at $94 \mathrm{GHz}$ with a much larger dataset embraced by a systematic study of the dependence on labeling position, $n$, of the lipid chain, in cholesterol-containing membranes. ${ }^{7,8}$ We extend our previous work by including simulations for Brownian diffusion (as opposed to strong-jump) and compare the 94-GHz spectra with corresponding ones obtained with an EPR frequency of $9 \mathrm{GHz}$,

\footnotetext{
$\dagger$ Part of the special issue "Jack H. Freed Festschrift"

* To whom correspondence should be addressed. Tel.: +49-551 201 1285. Fax: +49-551 201 1501. E-mail: dmarsh@gwdg.de.

$\doteqdot$ Max-Planck-Institut für biophysikalische Chemie.

$\S$ Russian Academy of Sciences.
}

at low field. Essential aspects of the analysis are allowance for the polarity dependence of the nitroxide $g$ tensor at the measurement temperature of $30^{\circ} \mathrm{C}$ and investigation of possible residual effects that overall slow-motional components may have on the 94-GHz spectra (as opposed to higher-frequency spectra, e.g., at $250 \mathrm{GHz}$ ).

\section{Methods}

A. Simulations. Rapid Motion. Simulations using motional narrowing theory employ the model of Israelachvili et al., ${ }^{9}$ as depicted in Figure 1. In the Redfield limit, the spectra are independent of the mechanism for rotational diffusion (e.g., strong-jump, Brownian, etc.). The spin-label $z$ axis $\left(\mathbf{z}_{\mathrm{A}}\right.$, directed along the nitrogen $2 \mathrm{p} \pi$ orbital of the nitroxide) is parallel to the principal molecular axis of the spin-labeled segment for the 1-acyl-2-[n-(4,4-dimethyl-oxazolidine- $N$-oxy)] stearoyl-sn-glycero-3-phosphocholine ( $n$-PCSL) spin-labeled probes considered here (see e.g., ref 10). This latter axis performs limited angular fluctuations of amplitude $\theta_{\mathrm{A}}$ about the director (i.e., the membrane normal, $\mathbf{N})$. This off-axis motion is characterized by an order parameter $\left\langle\mathrm{P}_{2}\left(\cos \theta_{\mathrm{A}}\right)\right\rangle=1 / 2\left(3\left\langle\cos ^{2} \theta_{\mathrm{A}}\right\rangle-1\right)$, where angular brackets indicate an average over the $\theta_{\mathrm{A}}$ distribution, and by the rotation correlation time, $\tau_{\mathrm{R} \perp}{ }^{(A)}$. The spin label also performs limited random angular oscillations about its $z$ axis, that are independent of the orientation of the $z$ axis itself. This azimuthal $\phi$ oscillation is characterized by the pseudo-order parameter $\langle\cos 2(\phi-\bar{\phi})\rangle$, where $\bar{\phi}$ is the mean $\phi$-orientation and angular brackets indicate an average over the $\phi$ distribution, and by the rotational correlation time, $\tau_{\mathrm{R} \|}{ }^{(\mathrm{A})}$. This $\phi$ angle is defined by the nitroxide $x$ axis $\left(\mathbf{x}_{\mathrm{A}}\right.$, directed along the $\mathrm{N}-\mathrm{O}$ 


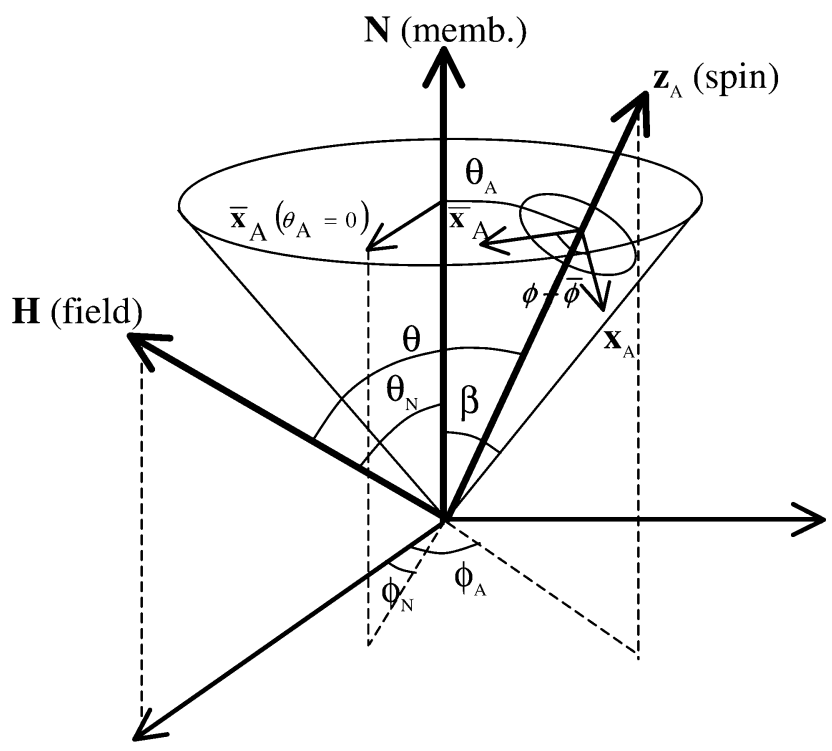

Figure 1. Relations between the instantaneous spin label $z$ axis $\left(\mathbf{z}_{A}\right)$, director axis (i.e., membrane normal, $\mathbf{N}$ ), and the laboratory magnetic field direction, $\mathbf{H}$. The nitroxide $z$ axis is assumed to coincide with the molecular long axis that has uniaxial ordering, relative to the director, $\mathbf{N}$. $\mathbf{z}_{\mathrm{A}}$ and $\mathbf{H}$ are inclined at $\theta_{\mathrm{A}}$ and $\theta_{\mathrm{N}}$, respectively, to $\mathbf{N}$, and $\mathbf{z}_{\mathrm{A}}$ is inclined at $\theta$, with azimuth $\phi_{\mathrm{A}}$ in the director system, to $\mathbf{H}$. The maximum amplitude of $\theta_{\mathrm{A}}$ is $\beta . \overline{\mathbf{x}}_{\mathrm{A}}$ is the direction of the mean nitroxide $x$ axis which performs $\phi$ rotations about $\underline{\mathbf{z}}_{\mathrm{A}}$, with mean value $\bar{\phi}$ about which the maximum amplitude is $\phi-\bar{\phi}=\phi_{0} . \phi_{\mathrm{N}}$ is the azimuthal orientation of the $\overline{\mathbf{x}}_{\mathrm{A}}$ axis for $\theta_{\mathrm{A}}=0$, in the director system.

bond), which has an azimuthal orientation $\phi_{\mathrm{N}}$ in the director axis system, when $\theta_{\mathrm{A}}=0$ (see Figure 1).

In the high-field approximation, the motionally averaged nitroxide spin Hamiltonian is given by ${ }^{7-9}$

$$
\begin{aligned}
& \left\langle\mathscr{H}_{\mathrm{s}}\left(\theta_{\mathrm{N}}, \phi_{\mathrm{N}}\right)\right\rangle= \\
& \left\{g_{0}+\frac{2}{3}\left\langle\mathrm{P}_{2}\left(\cos \theta_{\mathrm{A}}\right)\right\rangle \Delta g \mathrm{P}_{2}\left(\cos \theta_{\mathrm{N}}\right)+\frac{1}{6}\left[2+3\left\langle\cos \theta_{\mathrm{A}}\right\rangle+\right.\right. \\
& \left.\left.\left\langle\mathrm{P}_{2}\left(\cos \theta_{\mathrm{A}}\right)\right\rangle\right]\langle\cos 2(\phi-\bar{\phi})\rangle \delta g \sin ^{2} \theta_{\mathrm{N}} \cos 2 \phi_{\mathrm{N}}\right\} \beta_{e} H S_{z}+ \\
& \left\{a_{0}+\frac{2}{3}\left\langle\mathrm{P}_{2}\left(\cos \theta_{\mathrm{A}}\right)\right\rangle \Delta A \mathrm{P}_{2}\left(\cos \theta_{\mathrm{N}}\right)\right\} I_{z} S_{z}
\end{aligned}
$$

which describes the dependence of the motionally averaged resonances on the orientation $\left(\theta_{\mathrm{N}}, \phi_{\mathrm{N}}\right)$ of the magnetic field, $\mathbf{H}$, relative to the director, $\mathbf{N}$ (see Figure 1). The spin-Hamiltonian $g$ tensor is defined by

$$
\begin{aligned}
& g_{0}=\frac{1}{3}\left(g_{x x}+g_{y y}+g_{z z}\right) \\
& \Delta g=g_{z z}-\frac{1}{2}\left(g_{x x}+g_{y y}\right)
\end{aligned}
$$

and

$$
\delta g=\frac{1}{2}\left(g_{x x}-g_{y y}\right)
$$

Similar relations hold for the ${ }^{14} \mathrm{~N}$ hyperfine tensor, A. For simplicity, it is assumed that $\delta_{\mathrm{A}}=0 \mathrm{in}$ eq 1, appropriate to a purely dipolar ${ }^{14} \mathrm{~N}$-hyperfine anisotropy. For the purpose of simulation, this simplifying restriction is dropped, as, where appropriate, is the high-field approximation.

The resonance field position of hyperfine manifold $m_{\mathrm{I}}$ is given from eq 1 by

$$
\gamma_{\mathrm{e}} H_{\mathrm{m}_{\mathrm{I}}}\left(\theta_{\mathrm{N}}, \phi_{\mathrm{N}}\right)=\omega_{0} \frac{g_{0}}{g\left(\theta_{\mathrm{N}}, \phi_{\mathrm{N}}\right)}-m_{\mathrm{I}} A\left(\theta_{\mathrm{N}}\right)
$$

where $\gamma_{\mathrm{e}}$ is the electron gyromagnetic ratio, $\omega_{0}$ is the Larmor frequency corresponding to the isotropic $g$ value, $g_{0}$, and $m_{\mathrm{I}}$ is the ${ }^{14} \mathrm{~N}$ nuclear magnetic quantum number $\left(m_{\mathrm{I}}=0, \pm 1\right)$. The effective angular-dependent $g$ value, $g\left(\theta_{\mathrm{N}}, \phi_{\mathrm{N}}\right)$, and hyperfine splitting constant, $A\left(\theta_{\mathrm{N}}\right)$, are given respectively by the first and second terms in curly brackets on the right of eq 1 .

From eqs 1 and 5, it follows that resonance positions in the motionally averaged spectrum are characterized by $g$ - and $A$-tensor anisotropies that are given by

$$
\begin{gathered}
\langle\Delta g\rangle=\left\langle\mathrm{P}_{2}\left(\cos \theta_{\mathrm{A}}\right)\right\rangle \Delta g \\
\langle\delta g\rangle=\frac{1}{6}\left[2+3\left\langle\cos \theta_{\mathrm{A}}\right\rangle+\left\langle\mathrm{P}_{2}\left(\cos \theta_{\mathrm{A}}\right)\right\rangle\right]\langle\cos 2(\phi-\bar{\phi})\rangle \delta g
\end{gathered}
$$

and

$$
\langle\Delta A\rangle=\left\langle\mathrm{P}_{2}\left(\cos \theta_{\mathrm{A}}\right)\right\rangle \Delta A
$$

Note that the isotropic values $g_{o}$ and $a_{o}$ are independent of the motional state, and vary only with the polarity of the local environment (see e.g., ref 3).

Thus, for rapid motion, the hyperfine anisotropy depends only on the conventional order parameter $\left\langle\mathrm{P}_{2}\left(\cos \theta_{\mathrm{A}}\right)\right\rangle$ (insofar as $\delta A \approx 0)$. The $g_{x x}-g_{y y}$ anisotropy depends additionally on $\left\langle\cos \theta_{\mathrm{A}}\right\rangle$ and $\langle\cos 2(\phi-\bar{\phi})\rangle$, as seen from eq 7 . Therefore, to obtain the "order parameter" $\langle\cos 2(\phi-\bar{\phi})\rangle$ associated with the azimuthal rotation, a value is required for $\left\langle\cos \theta_{\mathrm{A}}\right\rangle$. This can be obtained if the orientation pseudopotential $U\left(\theta_{\mathrm{A}}\right)$ governing the off-axis motion is known.

The simplest model for motion of the nitroxide $\mathrm{z}_{\mathrm{A}}$ axis is a restricted random walk within a cone of half-angle, $\beta$ (see Figure 1). Each orientation is weighted by $\sin \theta_{\mathrm{A}}$ and the $z$ axis order parameter is given by

$$
\left\langle\mathrm{P}_{2}\left(\cos \theta_{\mathrm{A}}\right)\right\rangle=\frac{1}{2} \cos \beta(1+\cos \beta)
$$

whence $\beta$ may be obtained from eq 8 by using the experimental value of $\langle\Delta A\rangle$. Then the angular average required to interpret the $g$-value anisotropy is given in this model by

$$
\left\langle\cos \theta_{\mathrm{A}}\right\rangle=\frac{1}{2}(1+\cos \beta)
$$

A corresponding model for the azimuthal $\mathbf{x}_{\mathrm{A}}$-axis ordering is completely random $\phi$-angle fluctuations of maximum amplitude $\pm \phi_{0}$ about the mean value, $\bar{\phi}$, then

$$
\langle\cos 2(\phi-\bar{\phi})\rangle=\sin \phi_{0} \cos \phi_{0} / \phi_{0}
$$

In this simplified approach, the restricted angular motion is characterized completely by the independent maximum amplitudes, $\beta$ of the $z$-axis tilt and $\phi_{0}$ of the rotation or twist of the $x$ axis about the $z$ axis (see Figure 1).

Simulation with the rapid motional model requires not only the resonance positions but also the resonance line widths. The latter are given by the angular-dependent contribution to the transverse relaxation time, $T_{2, m_{\mathrm{I}}}\left(\theta_{\mathrm{N}}, \phi_{\mathrm{N}}\right)$, which from timedependent perturbation theory is 


$$
\frac{1}{T_{2, m_{\mathrm{I}}}\left(\theta_{\mathrm{N}}, \phi_{\mathrm{N}}\right)}=\gamma_{\mathrm{e}}^{2}\left\langle\left[H_{m_{\mathrm{I}}}\left(\theta_{\mathrm{N}}, \phi_{\mathrm{N}}\right)-\left\langle H_{m_{\mathrm{I}}}\left(\theta_{\mathrm{N}}, \phi_{\mathrm{N}}\right)\right\rangle\right]^{2}\right\rangle \tau_{\mathrm{R}}^{(\mathrm{A})}
$$

where $H_{m_{\mathrm{I}}}\left(\theta_{\mathrm{N}}, \phi_{\mathrm{N}}\right)$, given by eqs 1 and 5 , is the resonance position for the static field oriented at polar angles $\left(\theta_{\mathrm{N}}, \phi_{\mathrm{N}}\right)$, relative to the director. Angular brackets indicate averages over the spin label motion, as defined above. Higher-order angular averages are needed than for the resonance positions; these are given for the restricted random walk (i.e., cone) model by Israelachvili et al.. ${ }^{9}$ Finally, Lorentzian line shapes characterized by the appropriate values of $T_{2, m_{\mathrm{I}}}\left(\theta_{\mathrm{N}}, \phi_{\mathrm{N}}\right)^{-1}$ and intrinsic $T_{2}{ }^{0-1}$ are assigned to each resonance position, with Gaussian convolutions for inhomogeneous broadening, if required. For nonaligned samples, pseudo-powder patterns are generated by summing over all field orientations $\theta_{\mathrm{N}}$ and $\phi_{\mathrm{N}}$.

Brownian Diffusion: Stochastic Liouville Treatment. A rigorous treatment of the spin-label line-shape problem in the case of Brownian (i.e., small-step) diffusion has been given by Freed and co-workers in terms of the stochastic Liouville equation for the spin density matrix, $\rho$ (see ref 11 , for a review)

$$
\frac{\partial \rho(\Omega, t)}{\partial t}=-i[\mathscr{H}(\Omega), \rho(\Omega, t)]-\Gamma(\Omega) \rho(\Omega, t)
$$

where the spin Hamiltonian, $\mathscr{H}(\Omega)$, depends on the Euler angles, $\Omega$, characterizing the orientation of the principal axes of the magnetic $A$ and $g$ tensors, relative to the constant magnetic field. $\Gamma(\Omega)$ is a Markov operator for the rotational motion, which includes both internal and overall reorientations of the spinlabeled molecule. In systems such as membranes with molecular order, the Euler angles $\Omega$ relate the magnetic axes to the membrane director $\mathbf{N}$ (see Figure 1). The spin Hamiltonian, but not $\Gamma(\Omega)$, is then dependent on an additional set of Euler angles $\Psi$, which specify the orientation of the director to the static magnetic field. With the definitions from Figure 1, the orientation of the magnetic axes is given by $\Omega \equiv\left(\phi, \theta_{\mathrm{A}}, \phi_{\mathrm{A}}\right)$. For anisotropic Brownian rotational diffusion in an orientational potential, $U(\Omega)$, the symmetrized diffusion operator $\Gamma(\Omega)$ is given by ${ }^{11,12}$

$$
\Gamma(\Omega)=\mathbf{L} \cdot \mathbf{D}_{\mathrm{R}} \cdot \mathbf{L}+\mathbf{L} \cdot \mathbf{D}_{\mathrm{R}} \cdot \mathbf{L} \frac{U(\Omega)}{2 k T}-\mathbf{L} \frac{U(\Omega)}{2 k T} \cdot \mathbf{D}_{\mathrm{R}} \cdot \mathbf{L} \frac{U(\Omega)}{2 k T}
$$

where $\mathbf{L}$ is the operator which generates an infinitesimal rotation (formally equivalent to the dimensionless angular momentum operator) and $\mathbf{D}_{\mathrm{R}}$ is the rotational diffusion tensor.

In general, the orientational pseudopotential can be expanded in terms of Wigner rotation matrices, $\mathscr{D}_{\mathrm{K}, \mathrm{M}} \mathrm{L}(\Omega)$. For uniaxial systems, the terms up to second order are ${ }^{11}$

$$
U(\Omega)=\epsilon_{0} \mathscr{D}_{0,0}{ }^{2}(\Omega)+\epsilon_{2}\left[\mathscr{D}_{2,0}{ }^{2}(\Omega)+\mathscr{D}_{-2,0}{ }^{2}(\Omega)\right]
$$

where the $\epsilon_{i}$ represent the strength of the orienting potential. The first, and leading, term in eq 15 corresponds to the usual Maier-Saupe pseudopotential. In this formalism, the principal order parameter is given by

$$
S_{z z}=\left\langle\mathscr{D}_{0,0}{ }^{2}\right\rangle=\frac{\int \mathscr{D}_{0,0}{ }^{2}(\Omega) \exp [-U(\Omega) / k T] \mathrm{d} \Omega}{\int \exp [-U(\Omega) / k T] \mathrm{d} \Omega}
$$

where averaging is performed over all angular orientations, $\Omega$. Only the $\epsilon_{0}$ term in eq 15 contributes to this element of the order tensor. Note that, because eq 14 is uniaxial, the higher order terms in the orienting potential cannot give rise to $g_{x x}-$ $g_{y y}$ anisotropy. They are responsible for asymmetry in the ordering tensor, i.e., $S_{x x} \neq S_{y y}$ for ordering of the $x$ - and $y$-diffusion axes relative to the unique director. (It was confirmed by simulation that increasing $\epsilon_{2}$ from 0 to $8-10$, in both positive and negative directions, did not affect the $x-y$ averaging that is achieved for diffusion tensor values $\mathbf{D}_{\mathrm{R} \|} \geq 10^{8} \mathrm{~s}^{-1}$.)

For the oxazolidine phospholipid spin labels used here, the $\mathbf{z}_{\mathrm{A}}$ axis (see Figure 1) coincides with the symmetry axis of the molecular diffusion tensor. Thus, the above model accounts for unrestricted rotation of a spin label around its $z$ axis in the molecular frame and off-axial diffusion of this axis in an orienting potential in the director frame. Simulations according to this model are implemented with software by Freed and associates that is available from the Cornell EPR Centre. ${ }^{13,14}$ For nonaligned samples, simulated spectra are weighted by $\sin \theta_{\mathrm{N}}$ and integrated over the $\theta_{\mathrm{N}}$ angle.

Composite Motions on Different Time Scales. Studies on a spin-labeled lipid (16-PCSL) in membranes of dipalmitoyl phosphatidylcholine, with and without $50 \mathrm{~mol} \%$ cholesterol, have revealed that the high-field spectra at $250 \mathrm{GHz}$ are sensitive only to the rapid motions. ${ }^{6}$ The low-field spectra at $9 \mathrm{GHz}$, however, are additionally sensitive to a slow-motional component arising from overall lipid chain motion. Earlier studies at $9 \mathrm{GHz}$ employing motional models that specifically distinguish between segmental and overall chain motion also revealed that the former is much more rapid than the latter. ${ }^{15,16}$

Simulation of spectra from spin labels undergoing slow motion, in addition to a rapid motional component, can be achieved by using the stochastic Liouville equation with a spin Hamiltonian $\langle\mathscr{H}(\Omega)\rangle$ (see eq 1 ) that contains the $g$ and $A$ tensors that are partially averaged by the rapid motion. ${ }^{16}$ This approach is used here to simulate $9 \mathrm{GHz}$ spectra by using motionally averaged $g$ and $A$ tensors obtained from the $94 \mathrm{GHz}$ spectra. Simulations are again implemented by using the software described by Schneider and Freed ${ }^{13}$ with extensions by Budil et al. ${ }^{14}$

B. 9-GHz Spectroscopy. Low-field spectra were obtained on a Bruker EMX 9-GHz spectrometer operating with a rectangular cavity in the conventional continuous-wave mode. Fully hydrated samples were pelleted in 1-mm-diameter glass capillaries that were then accommodated in a 4-mm-diameter quartz EPR tube, which contained light silicone oil for thermal stability. Sample tubes were thermostated in a double-wall quartz dewar by a nitrogen gas-flow system. Sample temperature was measured with a fine-wire thermocouple in the silicone oil at the top of the cavity.

Otherwise, all details of the sample and sample preparation were as described previously for $94 \mathrm{GHz}$ measurements. ${ }^{7}$

C. Spectral Fitting. Fitting simulated spectra to the experimental high-field spectra was performed in two stages. The first criterion for fitting was coincidence of the resonance positions for all turning points of the simulated and experimental spectrum. Then the line widths were fitted, and after that, the resonance positions were again refined. These procedures were performed manually. It was found that least-squares optimization with respect to the first-derivative spectra sometimes resulted in unsatisfactory fits of the outer resonance positions, because of bias towards the larger central peaks.

For the lower frequency spectra, simulations with the software of ref 14 were repeated until coincidence of the resonance positions in the simulated and experimental spectra was achieved. Because of problems with local minima, simulations were checked by starting from different sets of input parameters. 

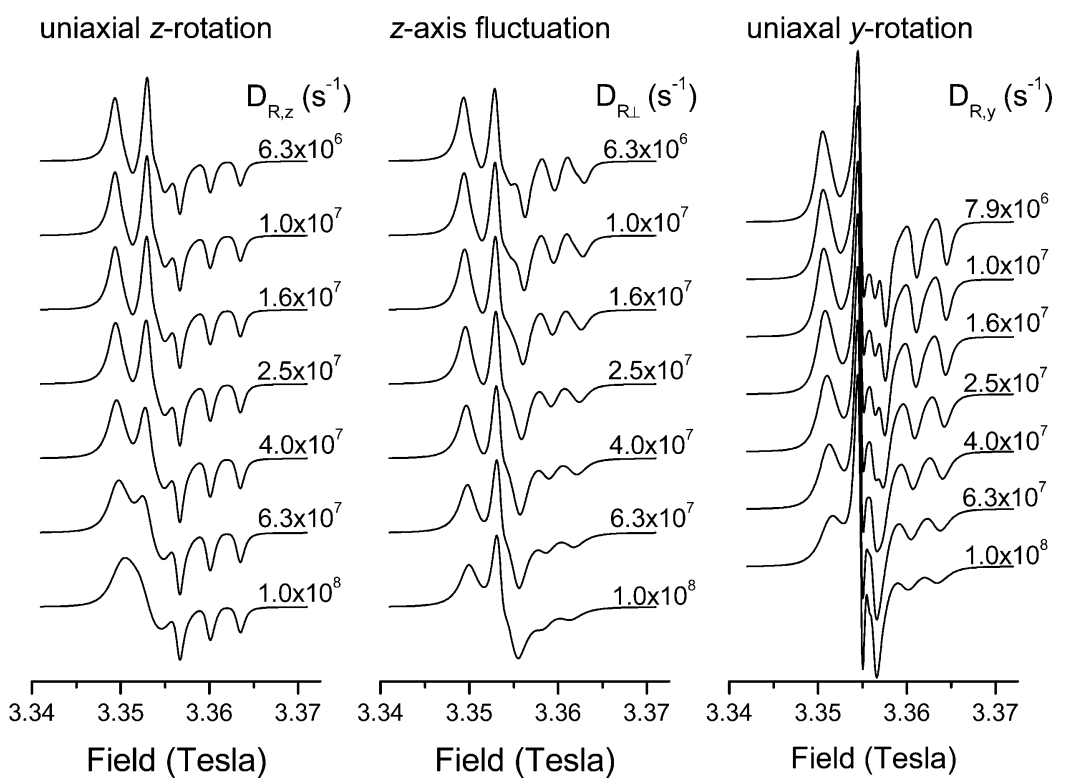

Figure 2. 94-GHz spin-label EPR spectra simulated using the stochastic Liouville equation and the Brownian diffusion model. Left-hand panel: spectra simulated for rotational diffusion solely about the nitroxide $z$ axis $\left(S_{z z} \approx 1\right)$, as a function of the rotational diffusion coefficient, $D_{\mathrm{R}, z}$. Centre panel: spectra simulated for off-axis rotational diffusion, in a fixed orientational potential corresponding to an order parameter of $S_{z z}=0.49(\beta=$ $52.5^{\circ}$ ), as a function of the rotational diffusion coefficient, $D_{\mathrm{R} \perp}$. Right-hand panel: spectra simulated for Brownian diffusion solely about the nitroxide $y$ axis $\left(S_{y y} \approx 1\right)$, given as a function of the rotational diffusion coefficient, $D_{\mathrm{R}, y}$. The rotational diffusion coefficient not being varied was fixed at $6.3 \times 10^{6} \mathrm{~s}^{-1}$. Simulation parameters are: tensors $g_{i i}=(2.0089,2.00585,2.00204)$ and $A_{i i}=(0.5,0.5,3.4)$ mT; Lorentzian line widths $\Delta H_{\mathrm{L}}=0.4,0.3$, and $0.3 \mathrm{mT}$ for $m_{\mathrm{I}}=+1,0$, and -1 ; and Gaussian broadening $\Delta H_{\mathrm{G}}=0.3 \mathrm{mT}$.

Approximate uncertainties in fitted parameters from $9 \mathrm{GHz}$ simulations are $1.5-2 \%$ for both $S_{z z}{ }^{\text {over }}$ and $D_{\mathrm{R} \perp}{ }^{\text {over }}$.

\section{Results}

Model simulations of high-field (i.e., $94 \mathrm{GHz}$ ) spin-label spectra have been presented previously for rapid motion, as have those for strong-jump diffusion. ${ }^{5}$ Here, in the first two sections, we present a theoretical simulation study of $94-\mathrm{GHz}$ spin-label spectra using the Brownian diffusion model. This is followed by analysis of experimental spectra from lipid membranes in which the nitroxide is at different segmental positions in the $s n-2$ acyl chain of a spin-labeled phospholipid. Part of the thrust of the analysis in the latter case is a multifrequency approach in which $94-\mathrm{GHz}$ spectra are used to obtain details of rapid segmental motions, and $9-\mathrm{GHz}$ spectra are used subsequently to obtain details of the slow, long-axis motion (cf. ref 6). Simulation of the experimental spectra must take into account the polarity dependence of the nitroxide $g$ tensor. This is done by comparing polarity profiles with spin-label position, $n$, that are obtained from determinations of $g_{0}$ (i.e., the trace of the $g$ tensor) at low temperature and at the actual measurement temperature.

A. High-Field Simulations for Anisotropic Brownian Rotation about Different Axes. Simulations of high-field spectra for spin labels undergoing Brownian (i.e., small-step) rotational diffusion are obtained from solution of the stochastic Liouville equation incorporating an orientational potential. Unlike in the rapid motional model, ${ }^{5}$ complete axial (i.e., azimuthal) rotation is assumed. Here we concentrate on slow rotational diffusion, such that the $x-y$ anisotropy is not completely averaged, and on rotation about different axes (II and $\perp$ to $\mathbf{z}_{\mathrm{A}}$ ).

Figure 2 (left-hand panel) gives 94-GHz spectra simulated for unrestricted rotation solely about the nitroxide $z$ axis. Spectra are given for increasing rotational diffusion coefficient, $D_{\mathrm{R}, z}$. As expected, the $z$ region of the spectrum is totally insensitive to the uniaxial diffusion. In the $x-y$ region of the spectrum, however, motional narrowing is observed at the higher rotational rates. As found previously for simulations with the strong jump model, ${ }^{5}$ comparison of the spectra shown for axial Brownian diffusion in the left-hand panel of Figure 2 with those for nonaxial motional narrowing given in Livshits and $\mathrm{Marsh}^{5}$ indicates that the two different mechanisms of $x-y$ averaging can be distinguished by the greater spectral broadening produced by increase in rotational rate than by increase in rotational amplitude.

Figure 2 (center panel) gives $94 \mathrm{GHz}$-spectra simulated for off-axis rotation of the nitroxide $z$ axis in an orienting potential $\left(S_{z z}=0.49\right)$, as a function of the rotational diffusion coefficient, $D_{\mathrm{R} \perp}$. This represents approximately the lowest order parameter for which the high-field (i.e., $A_{z z}$ ) hyperfine peaks can be resolved up to realistically high rotational diffusion coefficients $\left(D_{\mathrm{R} \perp} \sim 10^{8} \mathrm{~s}^{-1}\right)$. For $D_{\mathrm{R} \perp}<4 \times 10^{7} \mathrm{~s}^{-1}$, the principal change is seen in the $z$ region of the spectrum, on the high-field side. This consists of an increase in line width, accompanied by a downward shift in line position (see Figure 3, upper panel). These spectral features are characteristic of rotation in the slow motional regime (see e.g., ref 18 ). In the $x$ and $y$ regions of the spectrum, towards low field, the spectral changes for $D_{\mathrm{R} \perp}<4$ $\times 10^{7} \mathrm{~s}^{-1}$ are less than in the $z$ region. Only for $D_{\mathrm{R} \perp}>4 \times 10^{7}$ $\mathrm{s}^{-1}$ do the $x$ and $y$ spectral peaks broaden and the $x$ region move towards the $y$ position, displaying incipient $(x-y)$ motional narrowing. This indicates that the $x, y$ region of the spectrum lies deeper in the slow motional regime than does the $z$ region (cf. ref 18). This point will be returned to later, when simulating the experimental 94-GHz spectra from spin-labeled lipids.

Figure 2 (right-hand panel) gives 94-GHz spectra simulated for rotation solely about the (perpendicular) nitroxide $y$ axis. Spectra are given for increasing off-axial rotational diffusion coefficient, $D_{\mathrm{R}, y}$. With increasing rotational rate, the high-field $z$ region of the spectrum not only broadens but also moves as a whole to lower field. This again is characteristic of rotation in the slow motional regime. A remarkable feature, however, is that the motionally averaged hyperfine splitting, $\left\langle A_{z z}\right\rangle$, changes 


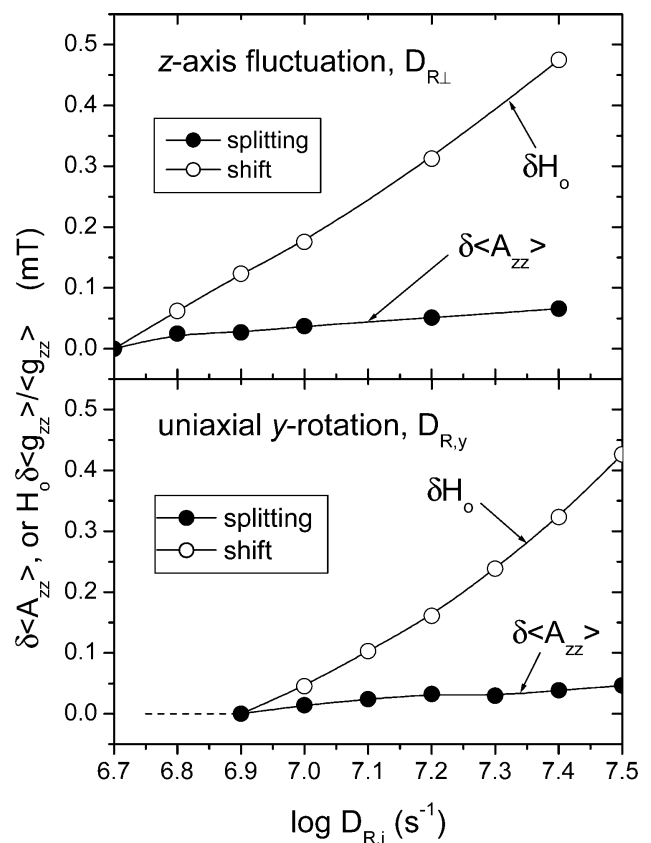

Figure 3. Upper panel ( $z$-axis fluctuation): Decrease in hyperfine splitting $\delta\left\langle A_{z z}\right\rangle$, and shift, $\delta H_{0}=H_{0} \delta\left\langle g_{z z}\right\rangle\left\langle g_{z z}\right\rangle$, of the central hyperfine manifold $\left(m_{\mathrm{I}}=0\right)$, in the $z$ region of the simulated $94-\mathrm{GHz}$ spin-label EPR spectra that are given in the center panel of Figure 2. Lower panel ( $y$-axis rotation): Decrease in hyperfine splitting $\delta\left\langle A_{z z}\right\rangle$ and shift, $\delta H_{0}$ $=H_{0} \delta\left\langle g_{z z}\right\rangle /\left\langle g_{z z}\right\rangle$, of the central hyperfine manifold $\left(m_{\mathrm{I}}=0\right)$, in the $z$ region of the simulated $94-\mathrm{GHz}$ spin-label EPR spectra that are given in the right-hand panel of Figure 2.

relatively little during this progression. The lower panel of Figure 3 shows that the hyperfine splitting decreases by only $\delta\left\langle A_{z z}\right\rangle=0.04 \mathrm{mT}$, whereas the absolute spectral shift of the centroid (i.e., $m_{\mathrm{I}}=0$ manifold) shifts by $H_{0} \delta\left\langle g_{z z}\right\rangle /\left\langle g_{z z}\right\rangle=0.4$ mT. A similar distinction is found also for $z$-axis fluctuation, in the upper panel of Figure 3. Therefore, although overall the 94-GHz spectra express some sensitivity to slow motion, the $\left\langle A_{z z}\right\rangle$ hyperfine splitting is sensitive only to fast motions. This simulation result forms an important basis for interpretation of the spectra at $94 \mathrm{GHz}$.

A further interesting feature that emerges from the slowmotion spectra shown in the right-hand panel of Figure 2 is the invariance of the trace of the effective $g$ tensor that is determined from the positions of the canonical $x, y$, and $z$ turning points. Over the whole range of $D_{\mathrm{R}, y}$, the $x$ and $z$ turning points can be discerned and the effective values of $g_{x x}, g_{y y}$, and $g_{z z}$ can be obtained by simulating the line shapes as pseudo-powder patterns. The shifts in the $x$ - and $z$-turning points increase by up to $1.12 \mathrm{mT}$ and $0.95 \mathrm{mT}$, respectively, on increasing $D_{\mathrm{R}, y}$ from $7.9 \times 10^{6}$ to $1 \times 10^{8} \mathrm{~s}^{-1}$, which correspond to changes in effective $g_{x x}$ by $6.75 \times 10^{-4}$ and in effective $g_{z z}$ by $5.75 \times$ $10^{-4}$. Nevertheless, the maximum change in the isotropic trace, $g_{0}$, is only about $1.5 \times 10^{-5}$. Thus, this parameter, viz., $g_{0}=$ $1 / 3\left(\left\langle g_{x x}\right\rangle+\left\langle g_{y y}\right\rangle+\left\langle g_{z z}\right\rangle\right)$, can be used for estimating the polarity from $94-\mathrm{GHz}$ spectra in the presence of molecular motion. For spin labels undergoing composite motion, the slow-motional component does not disturb determination of the true $g_{0}$ that is based on motional narrowing theory. This point will be returned to later for making corrections to the rigid-limit $g$ tensor determined at low temperature, to allow for the difference in environmental polarity of the spin labels at the actual temperature of measurement.

B. Rigid-Limit Spin Hamiltonian Tensors and Polarity Dependence. Spin Hamiltonian tensors for the $n$-PCSL spin labels in membranes of dimyristoyl phosphatidylcholine +40

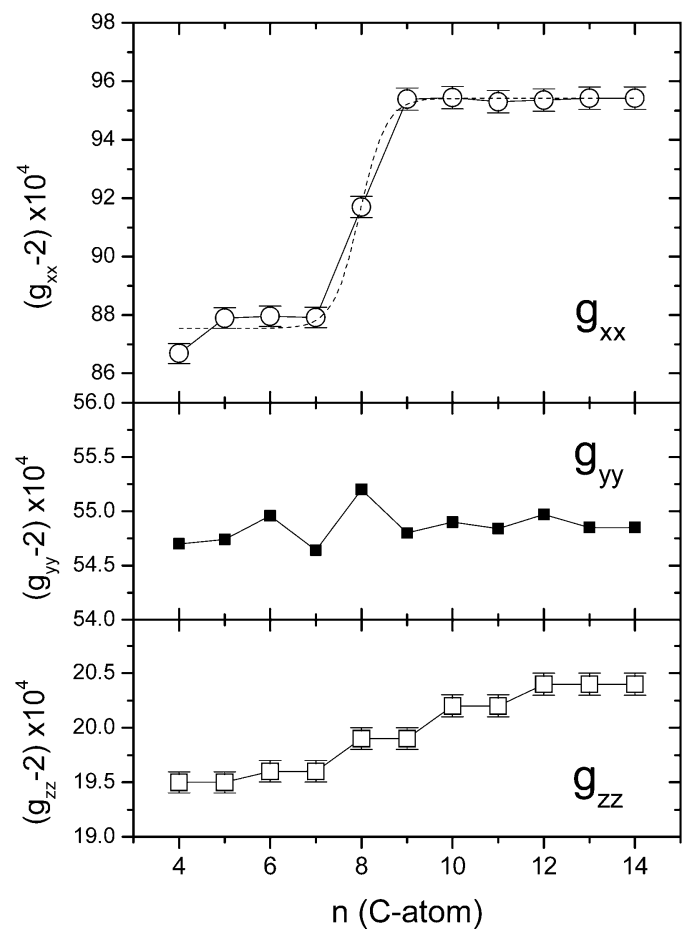

Figure 4. Dependence of the principal $g$-tensor elements, $g_{i i}$, on spinlabel position, $n$, in the $s n-2$ chain of $n$-PCSL in membranes of dimyristoyl phosphatidylcholine plus $40 \mathrm{~mol} \%$ cholesterol at -100 ${ }^{\circ} \mathrm{C}$. The dashed line in the upper panel is a nonlinear fit of eq 19.

mol \% cholesterol were obtained by simulating the powderpattern line shapes of rigid-limit $94-\mathrm{GHz}$ spectra obtained at $-100{ }^{\circ} \mathrm{C}$. Spectral data are from Kurad et al. ${ }^{17}$

Figure 4 gives the dependence of the $g$-tensor elements on spin-label position, $n$, at $-100{ }^{\circ} \mathrm{C}$. The $g_{x x}$ element displays a sigmoidal dependence on $n$ that represents the profile of local environmental polarity across the membrane thickness (see e.g. ref 3 ). The $g_{y y}$ and $g_{z z}$ elements change very little. For positions $n=4$ and 5 , the values of $g_{x x}$ are smaller than the partially motionally averaged values of $\left\langle g_{x x}\right\rangle$ obtained at the higher measurement temperature of $+30{ }^{\circ} \mathrm{C}$. This difference is diagnostic of a higher polarity for 4-PCSL and 5-PCSL in liquidordered membranes at $+30{ }^{\circ} \mathrm{C}$ than in frozen membranes at $-100{ }^{\circ} \mathrm{C}$. Motional effects at $+30^{\circ} \mathrm{C}$ can only tend to decrease $\left\langle g_{x x}\right\rangle$, as the $x$-turning point moves to higher field towards the canonical $y$ (and $z$ ) positions. This finding therefore shows that it is essential to correct the tensor values obtained at $-100{ }^{\circ} \mathrm{C}$ to the measurement temperature of $+30^{\circ} \mathrm{C}$, if simulations are faithfully to reflect the spin-label dynamics at the higher temperature.

Combined with the simulation results of the previous section, the trace of the $g$ tensor, i.e., $g_{0}$, affords a means to make this correction. Figure 5 compares the polarity profile for $g_{0}=1 /{ }_{3}\left(g_{x x}\right.$ $+g_{y y}+g_{z z}$ ) obtained from measurement at $-100{ }^{\circ} \mathrm{C}$ with that from $g_{0}=1 /{ }_{3}\left(\left\langle g_{x x}\right\rangle+\left\langle g_{y y}\right\rangle+\left\langle g_{z z}\right\rangle\right)$, which is obtained from simulations of the spectra obtained at $+30{ }^{\circ} \mathrm{C}$ that are described in the next section. Clearly, the polarity registered by $g_{0}$ is rather different for any given $n$-PCSL spin label in a membrane sample at the measurement temperature of $+30{ }^{\circ} \mathrm{C}$ from that in the frozen state at $-100^{\circ} \mathrm{C}$, where the rigid-limit spin Hamiltonian tensors are determined. The $g$-tensor elements and $A_{z z}$ value appropriate to $30^{\circ} \mathrm{C}$ can be obtained by using those determined at $-100{ }^{\circ} \mathrm{C}$ for the (different) positional isomer of $n$-PCSL that has the same value of $g_{0}$ as that determined for the positional isomer under question. This correspondence is readily deduced 


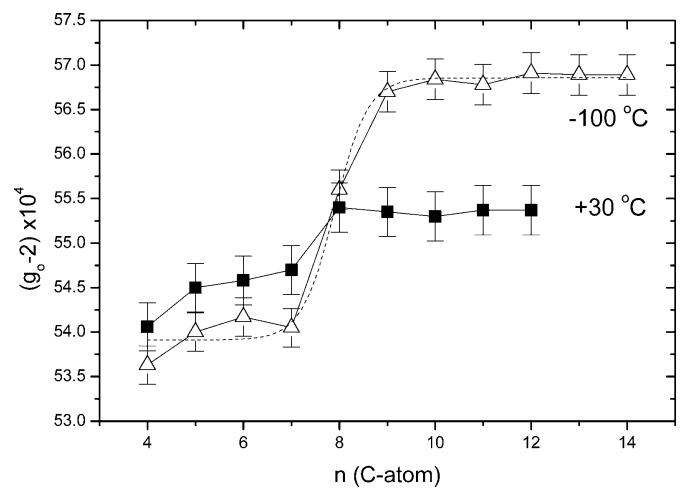

Figure 5. Dependence on spin-label position, $n$, in the $s n-2$ chain of $n$-PCSL in membranes of dimyristoyl phosphatidylcholine plus $40 \mathrm{~mol}$ $\%$ cholesterol of the $g$-tensor trace, $g_{o}$, calculated from the rigid-limit $g$-tensor elements at $-100{ }^{\circ} \mathrm{C}$ (triangles) and from the motionally averaged $\langle g\rangle$ tensor at $+30^{\circ} \mathrm{C}$ (squares). The dashed line is a nonlinear fit of eq 19 to the $-100{ }^{\circ} \mathrm{C}$ data.

TABLE 1: Polarity-Corrected Spin-Hamiltonian Tensor Elements of $\boldsymbol{n}$-PCSL Spin Labels in Membranes of Dimyristoyl Phosphatidylcholine +40 mol \% Cholesterol at $30{ }^{\circ} \mathrm{C}^{a}$

\begin{tabular}{ccccc}
\hline$n$-PCSL & $\mathrm{g}_{\mathrm{xx}}$ & $\mathrm{g}_{\mathrm{yy}}$ & $\mathrm{g}_{\mathrm{zz}}$ & $A_{z z}(\mathrm{mT})^{b}$ \\
\hline 4 & 2.00850 & 2.00586 & 2.00196 & 3.44 \\
5 & 2.00857 & 2.00586 & 2.00197 & 3.41 \\
6 & 2.00862 & 2.00585 & 2.00197 & 3.39 \\
7 & 2.00867 & 2.00585 & 2.00198 & 3.36 \\
8 & 2.00870 & 2.00585 & 2.00198 & 3.35 \\
9 & 2.00887 & 2.00583 & 2.00199 & 3.27 \\
10 & 2.00887 & 2.00582 & 2.00199 & 3.27 \\
11 & 2.00889 & 2.00587 & 2.00199 & 3.27 \\
12 & 2.00889 & 2.00587 & 2.00201 & 3.27 \\
13 & 2.00889 & 2.00587 & 2.00201 & 3.27 \\
14 & 2.00889 & 2.00587 & 2.00201 & 3.27
\end{tabular}

${ }^{a}$ Obtained from powder spectra at $-100{ }^{\circ} \mathrm{C}$ by selecting spin label positional isomers that have the same value of $g_{0}$ as that for the $n$-PCSL in question at $30{ }^{\circ} \mathrm{C}$ (see Figure 5 ). ${ }^{b} A_{x x}, A_{y y} \approx 0.5 \mathrm{mT}$ for all spin label positions.

from Figure 5. The resulting polarity-corrected spin-Hamiltonian tensor elements for $30^{\circ} \mathrm{C}$ are listed in Table 1 .

C. Simulation of High-Field Spectra from Lipid Labels in Membranes. Here we consider $94-\mathrm{GHz}$ spectroscopy of phosphatidylcholines ( $n$-PCSL) spin-labeled at different positions, $n$, of the $s n-2$ chain, in ordered-fluid lipid-bilayer membranes. Experimental data on $n$-PCSLs in membranes of dimyristoyl phosphatidylcholine that contain cholesterol have been presented elsewhere. ${ }^{7,8}$

The high-field spectra can be simulated reasonably adequately by using motional narrowing theory with the single-component model of Israelachvili et al..$^{9}$ for restricted azimuthal $\phi$ rotation about the nitroxide $z$ axis (cf. ref 5). Representative experimental and simulated 94-GHz spectra are given in Figure 6. Just as in the case for simulations with the strong-jump model, ${ }^{5}$ simulations for Brownian rotational diffusion with unrestricted $\phi$ rotation describe the experimental line shapes less well than do the spectra simulated with motional narrowing theory that are given in Figure 6 (data not shown). This result is in agreement with the findings of Lou et al. ${ }^{6}$ that high-field spectra are sensitive only to rapid motions of the lipid chains. The latter result was obtained at $250 \mathrm{GHz}$ and for a position of chain labeling close to the terminal methyl group. Spectra recorded at $94 \mathrm{GHz}$ may still display some residual sensitivity to slow overall motion of the lipid chains. This point is addressed in

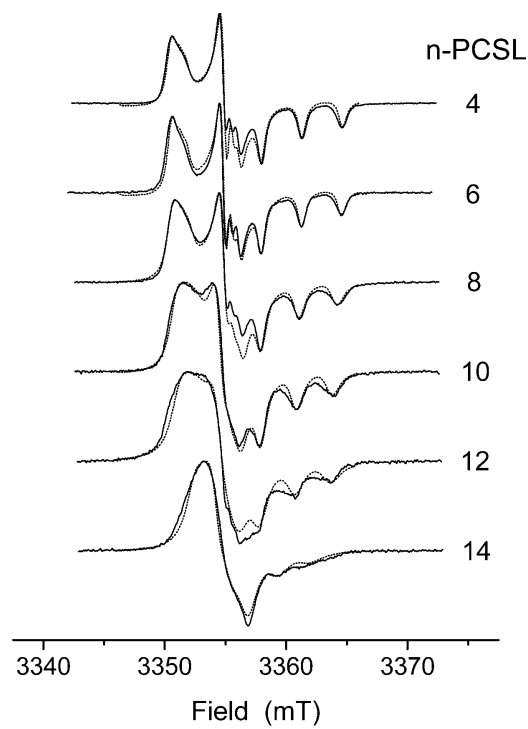

Figure 6. Solid lines: 94-GHz EPR spectra of $n$-PCSL spin-labels (1-acyl-2-[n-(4,4-dimethyloxazolidine- $N$-oxyl)] stearoyl-sn-glycero-3phosphocholine) in fully hydrated membranes of dimyristoyl phosphatidylcholine plus $40 \mathrm{~mol} \%$ cholesterol at $30{ }^{\circ} \mathrm{C}$. ${ }^{7,8}$ Spin-label position, $n$, in the lipid chain is indicated on the figure. Dashed lines: spectral simulations using motional narrowing theory, as described in the text, with rigid-limit spin Hamiltonian tensors corrected to $30^{\circ} \mathrm{C}$.

the next paragraph by using the results from slow-motional Brownian simulations that were described in the previous section.

The $z$ components of both the $\langle A\rangle$ and $\langle g\rangle$ tensors are quite generally insensitive to axial rotation on any time scale, unless the $z$-axis ordering is very low. As demonstrated by simulations for Brownian diffusion already given (see Figure 3), the value of $\left\langle A_{z z}\right\rangle$ (but not necessarily of $\left\langle g_{z z}\right\rangle$ ) at $94 \mathrm{GHz}$ is also relatively insensitive to slow off-axial diffusion. Thus, apart from polarity, $\left\langle A_{z z}\right\rangle$ is primarily sensitive only to the rapid, i.e., segmental, off-axial rotations, which can be treated by motional narrowing theory. A useful parameter for analyzing the rapid motions is the ratio

$$
\rho_{A}=\frac{\left\langle A_{z z}\right\rangle-\frac{1}{2}\left(A_{x x}+A_{y y}\right)}{A_{z z}-\frac{1}{2}\left(A_{x x}+A_{y y}\right)}
$$

This is related to the order parameter $\left\langle P_{2}\left(\cos \theta_{\mathrm{A}}{ }^{\mathrm{loc}}\right)\right\rangle$ that characterizes the local off-axis motions by

$$
\left\langle\mathrm{P}_{2}\left(\cos \theta_{\mathrm{A}}^{\mathrm{loc}}\right)\right\rangle=\frac{1}{2}\left(3 \rho_{\mathrm{A}}-1\right)
$$

which can be derived from eq 8 , together with the invariance of the trace of the $A$ tensor.

Table 2 compares the ratios, $\rho_{\mathrm{A}}$, obtained from the experimental values of $\left\langle A_{z z}\right\rangle$, with the corresponding ones $\left(\rho_{\mathrm{g}}\right)$ derived from the values of $\left\langle g_{z z}\right\rangle$. One sees that $\rho_{\mathrm{g}} \approx \rho_{\mathrm{A}}$ for $n=4-8$, but for larger values of $n$, the value of $\rho_{\mathrm{g}}$ tends to be less than that of $\rho_{\mathrm{A}}$. This indicates that there are contributions to $\rho_{\mathrm{g}}$ from slow motional components, for spin-label positions $n \geq 9$. Also given in Table 2 are values of the order parameter, $\left\langle P_{2}\left(\cos \theta_{\mathrm{A}}{ }^{\text {loc }}\right)\right\rangle$, for the rapid off-axis diffusion that is derived from $\rho_{A}$ by using eq 18 .

Table 3 gives the values of $\langle\delta g\rangle$ characterizing $x-y$ averaging that are obtained from the rapid-motional simulations. Along with the values of $\beta^{\text {loc }}$ that are obtained from $\left\langle\mathrm{P}_{2}\left(\cos \theta_{\mathrm{A}}{ }^{\text {loc }}\right)\right\rangle$ by 
TABLE 2: Hyperfine, $\left\langle A_{z z}\right\rangle$, and $g$-Value, $\left\langle g_{z z}\right\rangle$ Anisotropy Ratios, $\rho_{\mathrm{A}}$ and $\rho_{\mathrm{g}}$, Respectively (See eq 17), for 1-Acyl-2-[n-(4,4'-dimethyl-oxazolidine- $N$-oxy) $]$ stearoylsn-glycero-3-phosphocholine (n-PCSL) in Dimyristoyl Phosphatidylcholine $+\mathbf{4 0}$ mol \% Cholesterol Membranes at $30^{\circ} \mathrm{C}$

\begin{tabular}{cccc}
\hline$n$-PCSL & $\rho_{\mathrm{A}}$ & $\rho_{\mathrm{g}}$ & $\left\langle\mathrm{P}_{2}\left(\cos \theta_{\mathrm{A}}{ }^{\text {loc }}\right)\right\rangle^{a}$ \\
\hline 4 & 0.96 & 0.97 & 0.94 \\
5 & 0.97 & 0.96 & 0.95 \\
6 & 0.97 & 0.96 & 0.96 \\
7 & 0.97 & 0.95 & 0.95 \\
8 & 0.94 & 0.94 & 0.92 \\
9 & 0.93 & 0.92 & 0.89 \\
10 & 0.91 & 0.90 & 0.87 \\
11 & 0.90 & 0.87 & 0.85 \\
12 & 0.90 & 0.88 & 0.85 \\
13 & 0.85 & 0.82 & 0.77
\end{tabular}

${ }^{a}$ Order parameter for the rapid local segmental motion derived from $\rho_{\mathrm{A}}$ by using eq 18 .

TABLE 3: $g$-Value Nonaxiality for 1-Acyl-2-[n-(4,4'-dimethyl-oxazolidine- $N$-oxy $)]$ stearoyl$s n$-glycero-3-phosphocholine (n-PCSL) in Dimyristoyl Phosphatidylcholine $+\mathbf{4 0}$ mol \% Cholesterol Membranes at $30{ }^{\circ} \mathbf{C}^{a}$

\begin{tabular}{rccccc}
\hline $\mathrm{n}$ & $\beta^{\text {loc }}$ & $\delta g \times 10^{4}$ & $\langle\delta g\rangle \times 10^{4}$ & $\langle\cos 2(\phi-\bar{\phi})\rangle^{b}$ & $\phi_{0}{ }^{\text {loc } c}$ \\
\hline 4 & $15^{\circ}$ & 13.2 & 13.1 & 1.0 & $0^{\circ}$ \\
5 & $14^{\circ}$ & 13.5 & 13.0 & 0.98 & $11^{\circ}$ \\
6 & $14^{\circ}$ & 13.8 & 12.8 & 0.94 & $17^{\circ}$ \\
7 & $14^{\circ}$ & 14.1 & 12.7 & 0.92 & $20^{\circ}$ \\
8 & $19^{\circ}$ & 14.3 & 12.9 & 0.93 & $19^{\circ}$ \\
9 & $22^{\circ}$ & 15.2 & 12.4 & 0.85 & $28^{\circ}$ \\
10 & $25^{\circ}$ & 15.2 & 10.8 & 0.74 & $37^{\circ}$ \\
11 & $26^{\circ}$ & 15.1 & 9.8 & 0.68 & $42^{\circ}$ \\
12 & $26^{\circ}$ & 15.1 & 9.6 & 0.67 & $43^{\circ}$ \\
13 & $33^{\circ}$ & 15.1 & 7.5 & 0.54 & $52^{\circ}$
\end{tabular}

${ }^{a} \delta g$ is the polarity-corrected rigid-limit value (see Table 1) and $\langle\delta g\rangle$ is the motionally averaged value at $30^{\circ} \mathrm{C}$. Uncertainties in simulated parameters are approximately, $\pm 0.5^{\circ}, \pm 0.1 \times 10^{-4}, \pm(0.01-0.02)$, and $\pm\left(1^{\circ}-2^{\circ}\right)$ for $\beta_{\mathrm{loc}}, \delta g$, and $\langle\delta g\rangle,\langle\cos 2(\phi-\phi)\rangle$, and $\phi_{0}{ }^{\text {loc }}$, respectively. ${ }^{b}$ Order parameter $\langle\cos 2(\phi-\bar{\phi})\rangle$ obtained from eqs 7 and 10 by using values of $\beta^{\text {loc }}$ obtained from $\left\langle P_{2}\left(\cos \theta_{\mathrm{A}}^{\text {loc }}\right)\right\rangle$ in Table 2. ${ }^{c} \phi_{0}{ }^{l o c}$ is the effective axial rotational amplitude from eq 11.

using eq 9, one can derive values for the "order parameter" $\langle\cos 2(\phi-\bar{\phi})\rangle$ that is associated with the azimuthal rotation about the $z$ axis. This is done by using eqs 7 and 10. The effective maximum amplitude, $\phi_{0}{ }^{\text {loc }}$, of the $\phi$ rotation is then obtained from eq 11 for the random fluctuation model. These are possibly upper estimates because no correction is made to the $g$ values for slow axial diffusion.

D. Simulation of Low-Field Spectra by Incorporating High-Field Results. Measurements at $94 \mathrm{GHz}$ therefore allow not only characterization of the rapid off-axis motion (from $\rho_{\mathrm{A}}$ ) but also to detect the onset of slow off-axis diffusional components (from comparison with $\rho_{\mathrm{g}}$ ). The latter is possible without resort to measurement at a second, lower frequency. Use additionally of spectra obtained at $9 \mathrm{GHz}$, however, improves precision in characterizing the slow off-axial component. From the values of $\left\langle\mathrm{P}_{2}\left(\cos \theta_{\mathrm{A}}{ }^{\mathrm{loc}}\right)\right\rangle$ that were obtained from $\rho_{\mathrm{A}}$, one can estimate the value of the $g$-tensor anisotropy $\langle\Delta g\rangle_{\text {loc }}$ that is averaged over the rapid off-axial motion. This is done by using eq 6 . Next, the value of $\langle\Delta g\rangle_{\text {loc }}$ obtained in this way, together with the experimental value of $\left\langle A_{z z}\right\rangle_{\text {loc }}$ from the $94 \mathrm{GHz}$ spectra, are used as input parameters for simulating the EPR spectra obtained at $9 \mathrm{GHz}$, which are dominated by the $z$ features. This is done by using the stochastic Liouville equation in which the tensors averaged by rapid motion, instead of the rigid limit values, are incorporated. These simulations

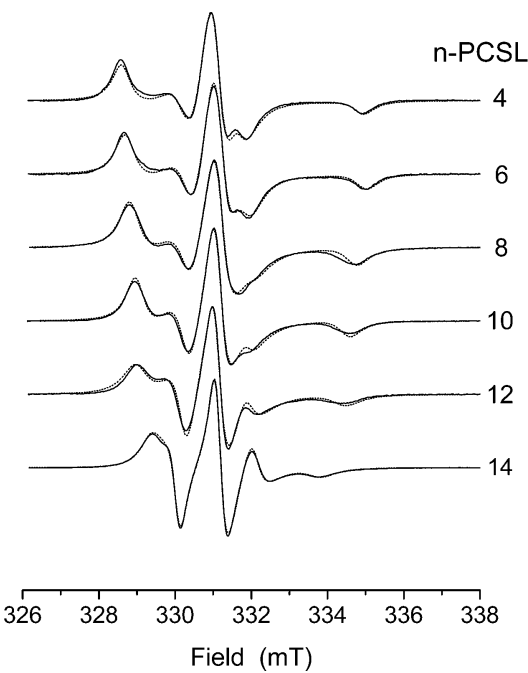

Figure 7. Solid lines: 9-GHz EPR spectra of $n$-PCSL spin-labels (1acyl-2-[n-(4,4-dimethyloxazolidine- $N$-oxyl)] stearoyl-sn-glycero-3-phosphocholine) in fully hydrated membranes of dimyristoyl phosphatidylcholine plus $40 \mathrm{~mol} \%$ cholesterol at $30^{\circ} \mathrm{C}$. Spin-label position, $n$, in the lipid chain is indicated on the figure. Dashed lines: spectral simulations for Brownian rotation using the stochastic Liouville formalism with motionally averaged spin Hamiltonian tensors obtained from the rapid-motion simulations of the $94-\mathrm{GHz}$ spectra given in Figure 6 . For further details see text.

TABLE 4: Parameters Governing the Slow Overall Motion of $n$-PCSL Spin Labels in Membranes of Dimyristoyl Phosphatidylcholine $+40 \mathrm{~mol} \%$ Cholesterol at $30{ }^{\circ} \mathrm{C}^{a}$

\begin{tabular}{rccc}
\hline$n$ & $S_{z z}{ }^{\text {over }}$ & $D_{\mathrm{R} \perp}{ }^{\text {over }}\left(\mathrm{s}^{-1}\right)$ & $D_{\mathrm{RII}}^{\text {over }}\left(\mathrm{s}^{-1}\right)$ \\
\hline 4 & 0.87 & $5.1 \times 10^{6}$ & $5.0 \times 10^{6}$ \\
5 & 0.83 & $5.1 \times 10^{6}$ & $1.4 \times 10^{7}$ \\
6 & 0.80 & $6.3 \times 10^{6}$ & $2.8 \times 10^{7}$ \\
7 & 0.83 & $6.3 \times 10^{6}$ & $2.8 \times 10^{7}$ \\
8 & 0.75 & $1.1 \times 10^{7}$ & $4.9 \times 10^{7}$ \\
9 & 0.61 & $8.9 \times 10^{6}$ & $4.9 \times 10^{7}$ \\
10 & 0.67 & $8.9 \times 10^{6}$ & $6.3 \times 10^{7}$ \\
11 & 0.66 & $1.0 \times 10^{7}$ & $5.6 \times 10^{7}$ \\
12 & 0.66 & $1.0 \times 10^{7}$ & $6.3 \times 10^{7}$
\end{tabular}

${ }^{a}$ Order parameter $\left(S_{z z}{ }^{\text {over }}\right)$ and anisotropic rotational diffusion coefficients $\left(D_{\mathrm{R} \|}{ }^{\text {over }}\right.$ and $\left.D_{\mathrm{R} \perp}{ }^{\text {over }}\right)$ are determined by simulation of $9-\mathrm{GHz}$ spectra using motionally averaged $g$ and $A$ tensors from $94-\mathrm{GHz}$ spectra, as described in the text.

then yield values of $D_{\mathrm{R} \perp}{ }^{\text {over }}$ and $S_{z z}{ }^{\text {over }}$ for the slow-motional component. It is significant to note that, at high field, the values of $\left\langle A_{z z}\right\rangle$ for the $n$-PCSL at $30{ }^{\circ} \mathrm{C}$ are appreciably higher than those at $9 \mathrm{GHz}$. The difference is about $0.15 \mathrm{mT}$ for $n=4-7$ and gradually increases for higher $n$. For rapid rotation, the motionally averaged values should be equal at both EPR frequencies. Thus the difference in $\left\langle A_{z z}\right\rangle$ is diagnostic for the presence of a slower diffusional motional mode that is off-axial in nature.

Figure 7 gives experimental spectra recorded at an EPR frequency of $9 \mathrm{GHz}$ (solid lines). Simulations with the motionally averaged spin-Hamiltonian tensors obtained from the rapidmotional simulations of the $94-\mathrm{GHz}$ spectra that are shown in Figure 6 are given by the dashed lines in Figure 7. The 9-GHz spectra are simulated adequately by this procedure, which (as already noted) they would not be by using simply the same simulation procedure and parameters as in the $94-\mathrm{GHz}$ case. Order parameters $\left(S_{z z}{ }^{\text {over }}\right)$ and diffusion coefficients $\left(D_{\mathrm{R} I}{ }^{\text {over }}\right.$ and $\left.D_{\mathrm{R} \perp}{ }^{\text {over }}\right)$ for the overall motion that are obtained by fitting the 9-GHz spectra are given in Table 4. The low values of the diffusion coefficients confirm that the overall motion of the lipid chain is in the slow regime at $9 \mathrm{GHz}$ and would have relatively minor influence on the spectra at $94 \mathrm{GHz}$. Attempts have been 
made to optimize the motional parameters by fitting the 9- and 94-GHz spectra iteratively using the simulation program of Schneider and Freed $^{13}$ (see ref 19). These are hampered as regards the $94-\mathrm{GHz}$ spectra, however, by the limitation of unrestricted $\phi$ rotation in the latter simulation model, which results in inadequate fits in the $x-y$ region of the spectra. Nevertheless, consensus fits can be obtained in the $z$ region of the spectrum.

\section{Discussion}

A. Model Simulations. The model simulations presented in Figure 2 establish two important principles for the interpretation of 94-GHz EPR spectra of spin-labeled lipids in membranes. Although these simulated spectra demonstrate some residual sensitivity to slow motion, both the ${ }^{14} \mathrm{~N}$ hyperfine splitting, $A_{z z}$, and the trace of the $g$ tensor, $g_{0}$, are insensitive to slow rotational motions. This means that the $A_{z z}$ hyperfine splitting at $94 \mathrm{GHz}$ is sensitive only to the fast components of composite motional modes.

A qualitative explanation of the latter result is as follows. At $94 \mathrm{GHz}$, all three $\left(m_{\mathrm{I}}=0, \pm 1\right)$ hyperfine components for the $z$-orientation shift in the same direction towards the $y$-and $x$-canonical positions. Therefore, the motional effect on the difference in line-shift of these components is, to a considerable extent, canceled out. These differences are further compensated because of the pseudosecular interaction $\left(S_{z} I_{ \pm}\right)$, which is equivalent to exchange between the hyperfine components. This unique situation at high field is in contrast to that familiar at the conventional EPR operating frequency of $9 \mathrm{GHz}$. In the latter case, the two outer hyperfine manifolds $\left(m_{\mathrm{I}}= \pm 1\right)$ at the $z$-position shift in opposite directions, approaching one another, as they move towards the $y$ - and $x$-canonical components that are located in the central part of the spectrum. Consequently, the value of $\left\langle A_{z z}\right\rangle$ for $9 \mathrm{GHz}$ spectra is extremely sensitive to off-axis rotation (as is well-known), whereas that at $94 \mathrm{GHz}$ and higher EPR frequencies is relatively insensitive to slow offaxial diffusion. Note that, in both regimes of operating frequency, the $z$ region of the spin-label EPR spectra remains relatively insensitive to axial rotation (see Figure 2, left-hand panel, for $94 \mathrm{GHz}$ ).

The invariance of the trace of the $g$ tensor at $94 \mathrm{GHz}$ means that this still may be used as an indicator of environmental polarity, even in the presence of slow motions. As already explained in the Results section, this simulation result affords a means for correcting the rigid limit spin Hamiltonian tensors obtained in the frozen state at low temperature to the environmental polarity of the spin label in the fluid state at higher temperatures of measurement.

The validity of these principles for analysis of $94-\mathrm{GHz}$ spinlabel spectra extends practically over the regime for which the canonical turning points can be resolved in the experimental spectra. In particular, for the high-field $\left\langle A_{z z}\right\rangle$-hyperfine splitting, the center panel of Figure 2 demonstrates validity down to order parameters $S_{z z}=0.49$, which is much lower than those determined here for cholesterol-containing membranes (see Table 2). In membranes without cholesterol, hyperfine splittings are resolved only for order parameters comparable to, or greater than, that used in Figure 2 (see ref 5).

B. Polarity Profile. As is well-known from the solvent dependence of the $g$ tensor ${ }^{20}$ and anticipated theoretically, ${ }^{21}$ the $g_{x x}$ element is most sensitive to the transmembrane polarity profile (see Figure 4). The trough-like profile of $g_{x x}$ across the membrane can be fitted by a Boltzmann sigmoidal form (with reflection symmetry about the membrane midplane) that was introduced previously to describe transmembrane polarity profiles registered by the isotropic ${ }^{14} \mathrm{~N}$ hyperfine coupling, $a_{0}{ }^{22}$

$$
g_{i}(n)=\frac{g_{i, 1}-g_{i, 2}}{1+\mathrm{e}^{\left(n-n_{0}\right) / \lambda}}+g_{i, 2}
$$

where $g_{i, 1}$ and $g_{i, 2}$ are the limiting values of $g_{i}$ at the polar headgroup and terminal methyl ends of the chain, respectively, $n_{0}$ is the value of $n$ at the point of maximum slope, corresponding to $g_{i}\left(n_{0}\right)=(1 / 2)\left(g_{i, 1}+g_{i, 2}\right)$, and $\lambda$ is an exponential decay constant. Equation 19 thus represents a two-phase distribution between membrane regions with $n>n_{0}$ and $n<n_{0}$, where the free energy of transfer for water depends on the distance from the $n=n_{0}$ dividing plane. A nonlinear, least-squares fit of eq 19 to the $g_{x x}$ profile (where $i \equiv x x$ ) is given by the dashed line in the upper panel of Figure 4. This yields values of $n_{0}=7.96$ \pm 0.06 and $\lambda=0.29 \pm 0.11\left(R^{2}=0.993, N=11\right)$. As might be anticipated, similar values are obtained by fitting eq 19 to the $g_{0}$ profile at $-100{ }^{\circ} \mathrm{C}$ that is given in Figure 5 (where $i \equiv$ o): $n_{0}=7.9 \pm 0.1$ and $\lambda=0.3 \pm 0.1\left(R^{2}=0.991, N=11\right)$. The midpoint of the transition between regions of high and low polarity is comparable to that found from the $a_{0}$ profile in fluid membranes, but the width of the transition region is considerably narrower (cf. ref 22). This difference in width is probably a particular feature of frozen membranes.

C. High-Field Spectra: Fast Motion. The equality of the anisotropy ratios, $\rho_{\mathrm{A}}$ and $\rho_{\mathrm{g}}$, for $n=4-7$ in Table 2 shows that there is virtually no contribution to the shift of $\left\langle g_{z z}\right\rangle$ from slow overall off-axial tumbling, i.e., that $\left\langle\mathrm{P}_{2}\left(\cos \theta_{\mathrm{A}}{ }^{\text {over }}\right)\right\rangle \approx 1$ (or the overall motion is so slow as not to affect $\left\langle g_{z z}\right\rangle$ ), for labels in the upper part of the chain. This is attributable to the pronounced effect of cholesterol on chain ordering. In contrast, the slow overall off-axis diffusion becomes appreciable for chain segment positions $n \geq 8$. This represents a diminishing ordering effect of the rigid sterol nucleus, which itself extends approximately down to $n \approx 11$ of the lipid chains. ${ }^{7}$ Note that the ordering by the environment (i.e., the orientational pseudopotential) varies towards the chain end. This represents an increase in detail in describing the chain motion that is possible from high-field measurements, as compared with the model of a uniform chain-axis ordering used in the most sophisticated descriptions of $n$-PCSL spectra obtained at conventional EPR frequencies (cf. refs 15 and 16).

The values of $\left\langle\mathrm{P}_{2}\left(\cos \theta_{\mathrm{A}}{ }^{\mathrm{loc}}\right)\right\rangle$ given in Table 2 represent the order parameter for the rapid off-axis diffusion. This ordering profile approximately mirrors that of the appearance of appreciable slow off-axis diffusion that was deduced by comparing $\rho_{\mathrm{g}}$ with $\rho_{\mathrm{A}}$. In the upper parts of the chain, the segmental ordering is high, corresponding to effective maximum amplitudes of $\beta^{\text {loc }} \sim 14-15^{\circ}$ (see Table 3 ). Ordering towards the end of the chain decreases progressively with increasing $n$, reaching effective amplitudes of $\beta^{\text {loc }} \sim 30^{\circ}$.

The results presented in Table 3 show that the rapid segmental $\phi$ fluctuations around the chain axis are of limited amplitude. In the upper part of the chain, up to $\mathrm{C}-8$, the maximum angular excursions, $\phi_{0}{ }^{\text {loc }}$ and $\beta^{\text {loc }}$, of $\phi$ and $\theta_{\mathrm{A}}$ are very limited, $20^{\circ}$ or less. Beyond this, the $\phi$ amplitude increases steeply. At the terminal methyl region (C-14), full axial rotation of the chain segments is approximately achieved, i.e., $\phi_{0}{ }^{\text {loc }} \approx 90^{\circ}$. The $\theta_{\mathrm{A}}$ amplitude increases simultaneously in this region of the chain and reaches maximum amplitudes of $\beta^{\text {loc }} \approx 40^{\circ}$ towards the terminal methyl end (C-14) of the chain.

The profiles of lateral and transverse segmental chain ordering induced by cholesterol can be interpreted in terms of the shape, 
rigidity and flexibility of the various parts of the sterol molecule. This, and the possible implications of lateral chain ordering in membrane domain formation, are discussed by Kurad et al. ${ }^{7}$ (see also refs 3 and 10).

D. Low-Field Spectra: Slow Motion. As reported under Results, the values of $\left\langle A_{z z}\right\rangle$ measured at $9 \mathrm{GHz}$ are less than those obtained at $94 \mathrm{GHz}$. This indicates that the 9-GHz spectra are sensitive to slow off-axial modes of rotational diffusion to which the $94 \mathrm{GHz}$ spectra are relatively insensitive. Spectral simulation of the composite motion at $9 \mathrm{GHz}$ confirms this.

From Table 4 , it is seen that the order parameter, $S_{z z}{ }^{\text {over }}$, corresponding to the slow overall motional mode gradually decreases with increasing $n$ down the chain. This result was already anticipated from comparison of the $A_{z z^{-}}$and $g_{z z^{-}}$ anisotropy ratios, $\rho_{\mathrm{A}}$ and $\rho_{\mathrm{g}}$, of the $94-\mathrm{GHz}$ spectra. Data in Table 4 are restricted to the range $n=4-12$. This is because the $A_{z z}$-turning points in the 94-GHz spectra, which are required for the multifrequency analysis described above, are no longer resolved beyond this range, for $n=13$ and 14 (see Figure 6). In the latter cases, a different fitting procedure, similar to that employed by Lou et al., ${ }^{6}$ reveals a sharper drop in $S_{z z}{ }^{\text {over }}$ on going to $\mathrm{C}-13$ and $\mathrm{C}-14$ than that found at positions higher in the chain. Table 4 also shows that as the amplitude of the slow overall motion increases (i.e., $S_{z z}{ }^{\text {over }}$ decreases), so also does the corresponding rotational rate given by $D_{\mathrm{R} \perp}{ }^{\text {over }}$ (and also $D_{\mathrm{R} / I^{\text {over }}}$. As already mentioned, the overall motion of the chain axis cannot be represented simply as that of a rigid rod. This is not entirely surprising for a flexible chain and represents a varying effect of the chain environment on proceeding deeper into the membrane that can be probed only by the multifrequency approach.

\section{Conclusions}

Even at the lower end of the high-frequency range, $94-\mathrm{GHz}$ spectra of lipid spin labels are sensitive primarily to the rapid segmental motions of the lipid chains. This is especially the case for the off-axial component of the rotational diffusion that is reflected by the $\left\langle A_{z z}\right\rangle$ hyperfine splitting. In addition, the invariance of the $g$-tensor trace is largely preserved in the 94$\mathrm{GHz}$ spectra, despite residual slow-motional sensitivity. This simulation result is important both for investigating transmembrane polarity profiles and for making polarity corrections to the rigid-limit spin-Hamiltonian tensors. Not only do the 94$\mathrm{GHz}$ spectra allow investigation of rapid axial $\phi$ rotation but also combination with simulation of lower-frequency spectra allows a far more detailed and precise description of the slow off-axial components of the overall chain motion.

Acknowledgment. We thank Martin Fechner for porting the Cornell slow-motional simulation program to Linux. This work was supported by the Schwerpunktprogramm "High-field EPR" of the Deutsche Forschungsgemeinschaft.

\section{References and Notes}

(1) Smirnov, A. I.; Belford, R. L.; Clarkson, R. B. Spin Labeling. The Next Millenium. Biological Magnetic Resonance; Plenum Press: New York, 1998; Vol. 14, Chapter 3, pp 83-107.

(2) Borbat, P. P.; Costa-Filho, A. J.; Earle, K. A.; Moscicki, J. K. Freed, J. H. Science 2001, 291, 266-269.

(3) Marsh, D.; Kurad, D.; Livshits, V. A. Chem. Phys. Lipids 2002, 116, 93-114. 6396.

(4) Liang, Z. C.; Freed, J. H. J. Phys. Chem. B 1999, 103, 6384-

(5) Livshits, V. A.; Marsh, D. J. Magn. Reson. 2000, 147, 59-67. 11056

(6) Lou, Y.; Ge, M.; Freed, J. H. J. Phys. Chem. B 2001, 105, $11053-$

(7) Kurad, D.; Jeschke, G.; Marsh, D. Appl. Magn. Reson. 2001, 21, $469-481$.

(8) Kurad, D.; Jeschke, G.; Marsh, D. Biophys. J. 2004, 86, 264-271.

(9) Israelachvili, J.; Sjösten, J.; Eriksson, L. E. G.; Ehrström, M.; Gräslund, A.; Ehrenberg, A. Biochim. Biophys. Acta 1975, 382, 125-141.

(10) Gaffney, B. J.; Marsh, D. Proc. Natl. Acad. Sci. U.S.A. 1998, 95, 12940-12943.

(11) Freed, J. H. Spin Labeling, Theory and Applications; Academic Press Inc.: New York, 1976; pp 53-132.

(12) Favro, L. D. Fluctuation phenomena in solids; Academic Press: New York, 1965; pp 79-101.

(13) Schneider, D. J.; Freed, J. H. Spin-Labeling. Theory and Applications. Biological Magnetic Resonance; Plenum Publishing Corp.: New York, 1989; Vol. 8, pp 1-76.

(14) Budil, D. E.; Lee, S.; Saxena, S.; Freed, J. H. J. Magn. Reson. A 1996, 120, 155-189.

(15) Moser, M.; Marsh, D.; Meier, P.; Wassmer, K.-H.; Kothe, G. Biophys. J. 1989, 55, 111-123.

(16) Cassol, R.; Ge, M. T.; Ferrarini, A.; Freed, J. H. J. Phys. Chem. B 1997, 101, 8782-8789.

(17) Kurad, D.; Jeschke, G.; Marsh, D. Biophys. J. 2003, 85, 10251033.

(18) Mason, R.; Freed, J. H. J. Phys. Chem. 1974, 78, 1321-1323.

(19) Livshits, V. A.; Marsh, D. Very high frequency (VHF) ESR/EPR. Biological Magnetic Resonance; Kluwer Academic: New York, 2004; Vol. 2 , in press.

(20) Ondar, M. A.; Grinberg, O. Ya.; Dubinskii, A. A.; Lebedev, Ya. S. Sov. J. Chem. Phys. 1985, 3, 781-792.

(21) Kawamura, T.; Matsunami, S.; Yonezawa, T. Bull. Chem. Soc. Jpn. 1967, 40, 1111-1115.

(22) Marsh, D. Proc. Natl. Acad. Sci. U.S.A. 2001, 98, 7777-7782. 